\title{
Living Alone or Together During Lockdown: Association with Mood, Immune Fitness and Experiencing COVID-19 Symptoms
}

\author{
Pauline A Hendriksen' \\ Pantea Kiani ${ }^{1}$ \\ Johan Garssen ${ }^{1,2}$ \\ Gillian Bruce ${ }^{3}$ \\ Joris C Verster $\mathbb{D}^{1,4}$ \\ 'Division of Pharmacology, Utrecht \\ Institute for Pharmaceutical Sciences, \\ Utrecht University, Utrecht, 3584CG, the \\ Netherlands; ${ }^{2}$ Global Centre of \\ Excellence Immunology, Nutricia Danone \\ Research, Utrecht, 3584CT, the \\ Netherlands; ${ }^{3}$ Division of Psychology and \\ Social Work, School of Education and \\ Social Sciences, University of the West of \\ Scotland, Paisley, PAI 2BE, UK; ${ }^{4}$ Centre \\ for Human Psychopharmacology, \\ Swinburne University, Melbourne, \\ VIC3I22, Australia
}

\begin{abstract}
Purpose: The aim of this study was to evaluate whether or not living alone or together throughout the lockdown had an impact on mood, perceived immune fitness, as well as the presence and severity of COVID-19 symptoms.

Methods: $\mathrm{N}=505$ participants completed an online survey, which included questions on living situations, as well as mood, perceived immune fitness and COVID-19 symptom presence and severity. These factors were assessed retrospectively for the time periods before and during the COVID-19 pandemic.

Results: An overall decrease in mood was observed for both those living alone and together during the lockdown period. However, significantly larger increases in feelings of loneliness were observed for the group living alone. Furthermore, both groups reported decreases in perceived immune fitness, whereas only the group living alone reported a significant increase in COVID-19 symptom presence and severity. Moreover, significant correlations were found between perceived immune fitness, anxiety, and loneliness. These correlations were strongest in the group living alone. Lastly, positive correlations were found between perceived immune fitness and mood outcomes with being active, optimistic and the ability to cope with stress. Increased optimism, being more active and the adequate ability to cope with stress were associated with a reduced negative impact on perceived immune fitness.

Conclusion: The first COVID-19 lockdown period in the Netherlands was a challenging period for both people who lived alone and those living together, resulting in decreases in mood and poorer perceived immune fitness. Those living alone were, however, more heavily impacted by the lockdown restrictions. This was further reflected by the increased presence and severity of COVID-19 symptoms in people who lived alone during the first COVID-19 lockdown in The Netherlands.
\end{abstract}

Keywords: corona virus, living situation, health, stress, quality of life

\section{Introduction}

On the 27th of January, the first 2019 coronavirus disease (COVID-19) infection was reported in the Netherlands. Due to the contagious nature of the virus, many restrictions were implemented to reduce the spread of the disease. The first lockdown was instigated from the 15th of March until the 11th of May 2020. Closure of many establishments, such as schools, restaurants and theatres, was enforced, where possible people had to work from home, and it was advised to stay-at-home as much as possible. ${ }^{1}$

Despite the effectiveness of measures aiming to control the spread of the virus, studies have shown that the COVID-19 pandemic and associated restrictions have
Correspondence: Joris C Verster Division of Pharmacology, Utrecht Institute for Pharmaceutical Sciences, Utrecht University, Universiteitsweg 99 Utrecht, 3584CG, the Netherlands Tel +31302536909

Email J.c.verster@uu.nl 
had a great impact on mental health. ${ }^{2}$ Following the onset of the pandemic, an overall pattern of increases in stress, loneliness and anxiety was observed. ${ }^{3}$ This may be due to ambivalence about the course of the pandemic, and various related factors such as fear of infection, and economic uncertainty. ${ }^{4,5}$ Furthermore, feelings of loneliness may increase as social contact with other decreases. Feelings of disconnection and isolation from others due to lack of social contact can further aggravate decreases in mood, particularly feelings of loneliness, which is linked to feelings of anxiety, depression, and stress. ${ }^{6-10}$

Literature reveals a large range of responses to the lockdown, and that it was not associated with the same level of negative mood effects for everyone. ${ }^{5}$ For example, differential effects have been reported according to age and sex, as well as for other demographic variables. For instance, women as well as people under the age of 40 were found to have higher rates of anxiety than men and those above the age of 40. Furthermore, women were found to have higher levels of psychological distress, as well as being more vulnerable to stress. ${ }^{8,11-14}$

Not only have studies shown that the pandemic has had negative effects on mental health but physical consequences have also been noted. Psychological stress can lead to the release of the corticotropin-releasing hormone, activating the hypothalamic-pituitary-adrenal (HPA)-axis. This results in increased levels of cortisol, the body's primary stress hormone. Increases in amounts hereof can trigger the development of stress-induced depression and anxiety. Although the body has a natural feedback mechanism, which uses cortisol to regulate its own production, it is found that social factors, such as social isolation and loneliness, can lead to glucocorticoid resistance, restricting the body's ability of maintaining this negative feedback system. ${ }^{15,16}$ Moreover, loneliness has also been associated with decreases in amounts of neutrophil to monocyte ratios as well as neutrophil to lymphocyte ratios. These effects have been observed to be in direct proportion to circulating cortisol levels, suggesting a relationship between stress and decreased immune functioning. ${ }^{6}$

Loneliness plays a key role in both the mental and physical wellbeing of a person.

Studies conducted in the UK, the US, Spain and Singapore, which looked at those living alone during the COVID-19 pandemic, demonstrate a correlation between living alone and an increased risk for feelings of loneliness. ${ }^{13}$ These studies confirm that living alone is a predisposing risk factor for feelings of loneliness, stress, anxiety and depression. ${ }^{8-10,17}$

Interestingly, literature analyzing those living together during the pandemic found mixed results, stating some flourish during this time, whereas others found the situation to bring additional stress into the household. ${ }^{18}$ This may be because the lockdown conditions are unique and different to "normal" pre-COVID life (eg, working from home, losing one's job, teaching children at home, and small housing).

A Turkish study indicated that living with others during this time may increase the risk of negative psychological effects such as increased stress. ${ }^{19}$ Furthermore, some may avoid close contact with people living together, perhaps related to the fear of COVID-19. ${ }^{20}$ This further posed as an indicator of increased feelings of loneliness. Moreover, an Australian study demonstrated that living with family members during the lockdown period can negatively impact daily life and mood. ${ }^{18}$ Many routine activities were altered during lockdown. Some parents were forced to work from home, as well as help their children with schoolwork, changing the nature of their parenting, and potentially placing unprecedented strain on parent-child relationships. $^{18}$

There are also several articles that highlight the benefits of living together during this period. An Italian study indicated that those living with children or their partners were more likely to experience positive emotions during the lockdown period. It further noted that increased interpersonal relationships with people living together had a positive effect on wellbeing. Bonds between people living together are important factors that modulate feelings of stress and other negative emotions. ${ }^{21}$ Furthermore, those living with family members reported improved interaction and communication as well as improved conflict resolution and social support when facing stressful situations. Finally, an American study looking at 3253 respondents found that those who did not have close contact with people living together had almost twice the levels of loneliness compared with those having close contact. ${ }^{20}$ Thus, contacts with others are important.

Interpersonal connections and bonding are of importance to most people, whether it be with significant others (friends and family) or through professional contacts. ${ }^{22}$ Studies have shown that individuals with a larger network of social relationships indicate higher quality of life as relationships bring with them a sense of belonging, as well as an environment in which one can trust each other and 
share problems. ${ }^{23}$ Furthermore, people may not only rely on others for moral and physical support but also count on others during times of despair, such as being ill. ${ }^{7}$ Consequently, it has been found that social support, resulting from strong interpersonal connections, can reduce negative mood. Social support has a stress-buffering effect and can alleviate feelings of depression, anxiety and distress, as well as being protective against feelings of loneliness. ${ }^{10,24}$

The current study investigated the impact of living alone or together during the first COVID-19 lockdown in the Netherlands. The study aimed to determine to what extent living alone or together had a differential effect on mood, perceived immune fitness and presence and severity of COVID-19 symptoms.

\section{Materials and Methods}

An online survey was conducted among Dutch adults, aged 18 years and older, between the 24th of June and the 26th of July $2020 .^{25}$ The survey was developed using SurveyMonkey and participants were recruited via Facebook. Ethics approval was obtained from the Ethics Committee of the Faculty of Social and Behavioral Sciences of Utrecht University (approval code FETC17061). The study was conducted in accordance with the Declaration of Helsinki, and electronic informed consent was obtained from all participants. A detailed description of the study methodology has been published elsewhere. ${ }^{25}$

Demographic data was collected, including whether participants lived alone or together with others during the COVID-19 lockdown. If they lived together with others, the number of people in the household was reported as well.

As a baseline health reference, immune status for 2019 (ie, before the COVID-19 pandemic) was assessed with the immune status questionnaire (ISQ). ${ }^{26}$ The ISQ consists of seven items, including "common cold", "diarrhea", "sudden high fever", "headache", "muscle and joint pain", "skin problems (eg, acne and eczema)", and "coughing". The items are scored on a 5-point Likert scale stating how often participants experienced these complaints during the past year, including "never", "sometimes", "regularly", "often", and "(almost) always". The overall ISQ score ranges from 0 (poor) to 10 (excellent), with higher scores indicating a better perceived immune fitness.

Other assessments were made for both the period before lockdown (15th of January - 14th of March 2020) and the lockdown period (15th of March - 11th of May 2020). Mood was assessed using single-item scales. ${ }^{27}$ The items included 'stress', "anxiety", "depression", "fatigue", "hostile", "loneliness" and "happy", and were scored on scales ranging from 0 (absent) to 10 (extreme). In a similar way, "being active" was assessed. Quality of life was assessed on a scale ranging from 0 (very poor) to 10 (excellent). ${ }^{27}$

Most of the countermeasures were assessed using the FANTASTIC Lifestyle Checklist. ${ }^{28,29}$ The original scale comprises 25 questions, and each item can be answered on a scale ranging from 0 to 4 . For the purpose of this study, the FANTASTIC Lifestyle Checklist was translated and modified by the authors. ${ }^{25}$ Sum-scores were computed for the domain supports of family and friends, physical activity level, nutrition, and coping with stress, whereas single item scores were used for the items sleep ("I sleep well and feel rested") and optimism ("I am a positive or optimistic thinker"). Higher scores on items or domains suggest a better or healthier lifestyle. Finally, "being active" was also assessed with a 1-item scale, ranging from 0 (extremely inactive) to 10 (very active).

Perceived immune fitness was assessed using a 1-item scale ranging from 0 (poor) to 10 (excellent), with higher scores indicating a better perceived immune fitness. ${ }^{30}$ The COVID-19 Symptoms Scale comprised 9 items, including sneezing, running nose, sore throat, cough, malaise/feeling sick, high temperature (up to 38 Celsius), fever (38 Celsius and higher), shortness of breath, and chest pain. ${ }^{25}$ The severity of each of the nine items could be rated as none (0), mild (1), moderate (2) or severe (3). The presence of COVID-19 symptoms was computed as the number of symptoms with a score $>0$. The COVID-19 severity score was computed as the sum score of all individual item scores, with a possible range from 0 (no complaints) to 27 (severe complaints).

Statistical analyses were conducted with SPSS (IBM Corp. Released 2013. IBM SPSS Statistics for Windows, Version 27.0. Armonk, NY: IBM Corp.). Mean and standard deviation (SD) were computed for all variables. Participants were allocated to a "live alone" or "live together" group. As the data were not normally distributed, non-parametric tests were used to compare the groups. Between-group comparisons were made using the Independent-Samples Mann-Whitney $U$-Test. Effect sizes were computed as $\eta^{2}=Z^{2} / n-1$.

Within group comparisons (ie, before lockdown versus during lockdown) were conducted using the Related 
Samples Wilcoxon Signed Rank Test. Effect sizes were computed as $\mathrm{ES}=\mathrm{Z} / \sqrt{ } 2 * \mathrm{n}$. Differences for mood and countermeasures were considered significant if $\mathrm{p}<0.00625$, after Bonferroni corrections for multiple comparisons, and if $\mathrm{p}<0.05$ for health correlates.

To further evaluate mood changes during lockdown, difference scores ( $\Delta$, during lockdown - before lockdown) were computed, and Spearman correlations were computed between the difference scores. Correlations were considered significant if $\mathrm{p}<0.00625$ for mood items (applying a Bonferroni correction for multiple comparisons) and $\mathrm{p}<0.05$ for other variables. Fisher $r-$ to- $z$ transformations were conducted, using an online calculator available at: http://vassarstats.net/rdiff.html, to compare the observed correlations of the "live alone" and "live together" groups. Differences between were considered significant if $\mathrm{p}<0.05$ (two-tailed).

The analyses were conducted for the sample as a whole, as well as for the potentially confounding factors sex and age. To examine the latter, participants were allocated to one of the following age groups: 18-35 years old, 36-65 years old, or $>65$ years old. Paired comparisons were conducted with the Kruskal-Wallis test, and differences were considered significant (after applying Bonferroni correction) if the adjusted $\mathrm{p}$ value was $<0.05$.

\section{Results}

$\mathrm{N}=505$ participants completed the survey, with an age range of 18 to 77 years old. Data on countermeasures (except "being active") were collected in Part 2 of the survey and completed by 264 participants (67 living alone and 197 living together with others). The mean (SD) number of household members was 2.8 (1.6), with a range from 1 to 12 . Their demographics are summarized in Table 1. No significant differences were observed between participants living alone or together.
Table 2 summarizes the mean (SD) of all study outcomes, assessed before and during the COVID-19 lockdown.

Table 2 shows that in both groups, mood was significantly poorer during lockdown compared to before lockdown. During the lockdown period, significant differences were observed between those who live alone and those living together with others.

Those living alone reported significantly higher scores for depression $\left(p<0.001, \eta^{2}=0.03\right)$, loneliness $\left(p<0.001, \eta^{2}=\right.$ 0.06 ), and significantly reduced ratings for quality of life $\left(p=0.004, \eta^{2}=0.02\right)$. It should be noted that before lockdown, significant differences already existed between the two groups for loneliness $\left(\mathrm{p}<0.001, \eta^{2}=0.05\right)$, depression $\left(p=0.002, \eta^{2}=0.02\right)$, and being happy $\left(p=0.002, \eta^{2}=0.02\right)$. In the group living alone, negative mood changes were accompanied by a significantly poorer perceived immune fitness $(p=0.009, E S=0.17)$, and a significant increase in reported COVID-19 symptom severity $(\mathrm{p}=0.024, \mathrm{ES}=0.15)$ and presence $(\mathrm{p}=0.045$, ES $=0.13)$. In the group living together, negative mood changes were also accompanied by a significantly poorer perceived immune fitness ( $p=0.013$, ES $=0.09$ ); however, no significant change was reported for the presence or severity of COVID-19 symptoms.

In addition to support from family and friends, in both groups, the other counter-measures were significantly less effective during lockdown compared to before lockdown. People that lived alone reported significantly less support from family and friends than those living together with others, both before lockdown $\left(\mathrm{p}<0.001, \eta^{2}=0.07\right)$ and during lockdown $\left(\mathrm{p}<0.001, \eta^{2}=0.06\right)$.

To further evaluate the relationship between the study outcomes, Spearman correlations of the difference scores (during lockdown - before lockdown) of mood, countermeasures, and health correlates with perceived immune fitness and the presence and severity of COVID-19

Table I Demographics

\begin{tabular}{|l|c|c|c|c|}
\hline & Overall & Living Alone & Living Together & p-value \\
\hline N (\%) & $505(100 \%)$ & $115(22.8 \%)$ & $390(77.2 \%)$ & - \\
Male/Female ratio & $174 / 331$ & $46 / 69$ & $128 / 262$ & 0.095 \\
Age (year) & $38.2(15.8)$ & $39.3(15.0)$ & $37.9(16.0)$ & 0.094 \\
Height (m) & $1.74(0.09)$ & $1.75(0.09)$ & $1.74(0.09)$ & 0.273 \\
Weight (kg) & $78.0(17.5)$ & $80.8(18.7)$ & $77.1(17.1)$ & 0.050 \\
BMI (kg/m²) & $25.7(5.2)$ & $26.4(5.4)$ & $25.5(5.2)$ & $0.05 I$ \\
ISQ & $7.0(2.3)$ & $7.2(2.1)$ & $7.0(2.4)$ & 0.598 \\
\hline
\end{tabular}

Notes: Mean and standard deviation (SD, between brackets) are shown.

Abbreviations: BMI, body mass index; ISQ, immune status questionnaire. 
Table 2 Assessments for Before and During Lockdown

\begin{tabular}{|c|c|c|c|c|}
\hline \multirow[b]{2}{*}{ Variables Assessed } & \multicolumn{2}{|c|}{ Living Alone } & \multicolumn{2}{|c|}{ Living Together With Others } \\
\hline & Before Lockdown & During Lockdown & Before Lockdown & During Lockdown \\
\hline \multicolumn{5}{|l|}{ Mood } \\
\hline Anxiety & I.7 (2.3) & $3.2(2.9) *$ & $1.5(2.2)$ & $2.5(2.7) *$ \\
\hline Depression & $2.2(2.5)$ & $3.4(3.1) *$ & $1.4(2.1)^{\dagger}$ & $2.3(2.8) * \dagger$ \\
\hline Loneliness & $2.7(2.5)$ & $4.6(3.3) *$ & $1.5(2.2)^{\dagger}$ & $2.7(2.9) * \dagger$ \\
\hline Fatigue & $3.9(2.7)$ & $4.5(2.8) *$ & $4.0(2.7)$ & $4.3(2.7)$ \\
\hline Hostile & $1.0(1.8)$ & $1.9(2.7) *$ & $0.8(1.6)$ & $1.4(2.3) *$ \\
\hline Happy & $6.7(2.0)$ & $5.7(2.3) *$ & $7.3(1.7)^{\dagger}$ & $6.3(2.1) *$ \\
\hline Stress & $3.5(2.7)$ & $4.4(2.9) *$ & $3.1(2.8)$ & $3.9(2.9) *$ \\
\hline \multicolumn{5}{|l|}{ Countermeasures } \\
\hline Optimism & $2.9(1.0)$ & $2.6(1.1) *$ & $3.2(0.9)$ & $2.9(1.1) *$ \\
\hline Coping with stress & $5.8(1.7)$ & $5.5(1.8) *$ & $6.0(1.8)$ & $5.8(1.9) *$ \\
\hline Support of family and friends & $5.4(1.9)$ & $5.6(1.8)$ & $6.5(1.8)^{\dagger}$ & $6.5(1.7)^{\dagger}$ \\
\hline Nutrition & $7.2(3.0)$ & $7.1(2.8)$ & $7.9(2.8)$ & $7.5(2.8) *$ \\
\hline Being active & $6.7(2.3)$ & $5.6(2.6) *$ & $6.9(2.3)$ & $5.7(2.5) *$ \\
\hline Physical activity level & $5.5(1.9)$ & $4.8(2.2)$ & $5.2(2.0)$ & $4.7(2.0) *$ \\
\hline Sleep & $2.8(1.0)$ & $2.6(1.1)$ & $2.8(1.0)$ & $2.7(1.0)$ \\
\hline \multicolumn{5}{|l|}{ Health correlates } \\
\hline Quality of life & $7.2(1.9)$ & $5.9(2.4) *$ & $7.6(1.6)$ & $6.6(2.0) * \dagger$ \\
\hline Perceived immune fitness & $7.5(1.6)$ & $7.1(1.8) *$ & $7.5(1.8)$ & $7.3(1.9) *$ \\
\hline COVID-19 Symptom presence & $2.5(2.2)$ & $2.9(2.2) *$ & $2.7(2.2)$ & $2.5(2.1)$ \\
\hline COVID-19 Symptom severity & $3.4(3.6)$ & $4.2(4.5) *$ & $3.7(3.8)$ & $3.6(3.8)$ \\
\hline
\end{tabular}

Notes: Mean and standard deviation (SD, between brackets) are shown. Differences for mood and countermeasures were considered significant if $\mathrm{p}<0.00625$, after Bonferroni correction for multiple comparisons, and if $p<0.05$ for health correlates. Significant differences between before and during lockdown are indicated by*; significant differences between the living alone group and living together group are indicated by ${ }^{\dagger}$.

symptoms were computed. The outcome of this analysis is summarized in Table 3.

The analysis revealed that all variables assessed correlated significantly with perceived immune fitness. This was evident in both groups, although the correlations of $\Delta$ perceived immune fitness with $\Delta$ anxiety and $\Delta$ loneliness were significantly stronger in the "living alone" group. The negative correlations suggest that increased negative mood is associated with poorer perceived immune fitness. In contrast, the positive correlations with countermeasures show that these assets improve perceived immune fitness. Although often statistically significant, the correlations between mood changes and the presence and severity of COVID-19 symptoms were less strong. The most robust correlations were found between optimism and the presence and severity of COVID-19 symptoms.

\section{Sex and Age Effects}

The data for men and women separately are summarized in Table 4.
The data of men and women followed the same pattern as the full sample in that for both sexes during lockdown mood was significantly poorer and quality of life was significantly reduced. No relevant differences between before and during lockdown were found for health correlates. For those living alone, before lockdown women reported significantly higher scores of fatigue and stress. During lockdown, no sex differences were found. For those living together with others, both before and during lockdown, women reported significantly higher scores of fatigue and stress compared to men. For both time periods, women also reported significantly poorer coping with stress than men, as well as a significantly lower perceived immune fitness.

The sample size is too small to divide the sample into age bins. Therefore, as an alternative, Spearman correlations were computed between age and the difference scores $(\Delta$, during lockdown - before lockdown) of mood, perceived immune fitness, and the presence and severity of COVID-19 symptoms. The analysis revealed significant correlations 
Table 3 Correlations with Perceived Immune Fitness and the Presence and Severity of COVID-19 Symptoms

\begin{tabular}{|c|c|c|c|c|c|c|}
\hline \multirow[b]{2}{*}{ Living Situation } & \multicolumn{2}{|c|}{$\begin{array}{c}\Delta \text { Perceived Immune } \\
\text { Fitness }\end{array}$} & \multicolumn{2}{|c|}{$\begin{array}{c}\Delta \text { COVID-19 Symptoms } \\
\text { Presence }\end{array}$} & \multicolumn{2}{|c|}{$\begin{array}{c}\Delta \text { COVID-19 Symptoms } \\
\text { Severity }\end{array}$} \\
\hline & Alone & Together & Alone & Together & Alone & Together \\
\hline \multicolumn{7}{|l|}{ Mood } \\
\hline$\Delta$ Anxiety & $-0.389 *$ & $-0.132^{\dagger}$ & 0.031 & 0.051 & 0.063 & 0.081 \\
\hline$\Delta$ Depression & $-0.407 *$ & $-0.262 *$ & 0.083 & $0.168 *$ & 0.037 & $0.179 *$ \\
\hline$\Delta$ Loneliness & $-0.381 *$ & $-0.126^{\dagger}$ & 0.060 & 0.108 & 0.053 & $0.140 *$ \\
\hline$\Delta$ Fatigue & $-0.338 *$ & $-0.241 *$ & $0.269 *$ & 0.104 & $0.265 *$ & 0.136 \\
\hline$\Delta$ Hostile & $-0.257 *$ & $-0.147 *$ & 0.063 & 0.031 & 0.041 & 0.034 \\
\hline$\Delta$ Happy & $0.364 *$ & $0.262 *$ & -0.113 & $-0.14 \mid *$ & -0.110 & $-0.159 *$ \\
\hline$\Delta$ Stress & $-0.293 *$ & $-0.263 *$ & 0.183 & 0.094 & 0.202 & 0.103 \\
\hline \multicolumn{7}{|l|}{ Countermeasures } \\
\hline$\Delta$ Optimism & $0.392 *$ & $0.147^{\dagger}$ & $-0.325 *$ & $-0.114^{\dagger}$ & $-0.288 *$ & -0.137 \\
\hline$\Delta$ Coping with stress & $0.419 *$ & $0.257 *$ & $-0.314 *$ & $-0.173 *$ & $-0.270 *$ & $-0.258 *$ \\
\hline$\Delta$ Support of family and friends & 0.071 & 0.049 & -0.069 & 0.000 & -0.046 & -0.014 \\
\hline$\Delta$ Nutrition & $0.355 *$ & 0.152 & -0.049 & 0.025 & -0.124 & -0.022 \\
\hline$\Delta$ Being active & $0.519 *$ & $0.318 * \dagger$ & 0.007 & $-0.100 *$ & -0.028 & -0.100 \\
\hline$\Delta$ Physical activity level & $0.356 *$ & $0.219 *$ & -0.117 & -0.173 & -0.120 & -0.180 \\
\hline$\Delta$ Sleep & $0.350 *$ & $0.226 *$ & -0.127 & -0.181 & -0.102 & $-0.235 *$ \\
\hline \multicolumn{7}{|l|}{ Health correlates } \\
\hline$\Delta$ Quality of life & $0.454 *$ & $0.267 * t$ & -0.080 & -0.083 & -0.073 & $-0.107 *$ \\
\hline$\Delta$ Perceived immune fitness & - & - & $-0.199 *$ & $-0.191 *$ & $-0.287 *$ & $-0.187 *$ \\
\hline$\Delta$ COVID-19 Symptom - presence & $-0.199 *$ & $-0.191 *$ & - & - & $0.890 *$ & $0.935 * \dagger$ \\
\hline$\Delta$ COVID-19 Symptom - severity & $-0.287 *$ & $-0.187 *$ & $0.890 *$ & $0.935 * \dagger$ & - & - \\
\hline
\end{tabular}

Notes: Notes: Spearman correlations are presented. Significant correlations are indicated by*. Significant differences between living alone and living together are indicated by $^{\dagger}$. Correlations were considered significant if $\mathrm{p}<0.00625$ for mood items (applying a Bonferroni correction for multiple comparisons) and $\mathrm{p}<0.05$ for other variables.

only between age and loneliness $(\mathrm{r}=-0.167, \mathrm{p}<0.001)$, age and hostility $(\mathrm{r}=0.168, \mathrm{p}<0.001)$, age and physical activity level $(\mathrm{r}=0.221 . \mathrm{p}<0.001)$, and age and nutrition $(\mathrm{r}=0.170$, $\mathrm{p}=0.006$ ). Thus, loneliness was experienced to a greater extent in younger participants, whereas older participants scored better on the countermeasures physical activity and nutrition. However, overall, the impact of sex and age on the study outcomes was small.

\section{Discussion}

Our findings show a significant decrease in overall mood for both groups following the onset of the lockdown. We show increases in feelings of anxiety, depression, loneliness, fatigue and hostility - results which are consistent with the current literature on mental health during the COVID-19 pandemic. ${ }^{2,10,21,31,32}$ These studies also found increased distress, anxiety, and depression during COVID-19, in particular in persons with poor coping strategies. $^{31,32}$ These negative mental health effects have been reported for the general population as a whole, as well as for specific subpopulations such as pregnant women and adolescents. ${ }^{33,34}$ This indicates that the lockdown period has had serious effects on the mental health of many, irrespective of their living situation. However, the effects were much more pronounced in those living alone compared to people that lived together with others.

Reasons for the observed mood changes may include anxiety and fear of COVID-19, as well as concerns about the wellbeing of others, such as friends or family members. ${ }^{35}$ Economic reasons may also play a role, such as worries or stress about unemployment rates and economic uncertainty. ${ }^{18}$ Furthermore, results indicate that people were less active during the lockdown period. Other studies previously found that a more sedentary lifestyle, together with reduced social opportunities, may contribute to people spending more time in bed and having poorer sleep quality, which may also impact mood. ${ }^{36}$ 
Table 4 Assessments for Before and During Lockdown, According to Sex

\begin{tabular}{|c|c|c|c|c|c|c|c|c|}
\hline \multirow{3}{*}{$\begin{array}{l}\text { Living Situation } \\
\text { Time Period } \\
\text { Sex }\end{array}$} & \multicolumn{4}{|c|}{ Living Alone } & \multicolumn{4}{|c|}{ Living Together With Others } \\
\hline & \multicolumn{2}{|c|}{ Before Lockdown } & \multicolumn{2}{|c|}{ During Lockdown } & \multicolumn{2}{|c|}{ Before Lockdown } & \multicolumn{2}{|c|}{ During Lockdown } \\
\hline & Men & Women & Men & Women & Men & Women & Men & Women \\
\hline \multicolumn{9}{|l|}{ Mood } \\
\hline Anxiety & $1.6(1.2)$ & $1.8(2.5)$ & $3.2(2.9) *$ & $3.1(2.9) *$ & $1.2(2.0)$ & $1.6(2.3)$ & $2.1(2.4) *$ & $2.7(2.9) *$ \\
\hline Depression & $2.3(2.5)$ & $2.1(2.5)$ & $3.5(3.3) *$ & $3.4(3.0) *$ & $1.1(1.9)^{\dagger}$ & $1.6(2.2)$ & $1.8(2.4) * t$ & $2.5(3.0) *$ \\
\hline Loneliness & $2.8(2.9)$ & $2.6(2.3)$ & $4.4(3.6) *$ & $4.7(3.0) *$ & $1.4(2.3)^{\dagger}$ & $1.6(2.2)^{\dagger}$ & $2.4(2.6) * t$ & $2.8(3.0) * t$ \\
\hline Fatigue & $2.9(2.5)$ & $4.5(2.6)^{\mathrm{S}}$ & $4.1(2.8) *$ & $4.9(2.9)$ & $3.4(2.6)$ & $4.4(2.7)^{\mathrm{s}}$ & $3.6(2.5)$ & $4.6(2.8)^{\mathrm{S}}$ \\
\hline Hostile & $1.2(1.9)$ & $0.8(1.8)$ & $2.4(2.9) *$ & $1.6(2.5) *$ & $0.8(\mathrm{I} .5)$ & $0.8(1.7)$ & $1.7(2.4) *$ & I.3 $(2.2) *$ \\
\hline Happy & $6.4(2.3)$ & $6.9(1.8)$ & $5.3(2.4) *$ & $6.0(2.3) *$ & $7.6(1.4)^{\dagger}$ & $7.1(1.8)$ & $6.6(2.0) * t$ & $6.1(2.2) *$ \\
\hline Stress & $2.6(2.4)$ & $4.1(2.7)^{\mathrm{S}}$ & $4.0(3.0) *$ & $4.7(2.8)$ & $2.4(2.6)$ & $3.4(2.8)^{\mathrm{S}}$ & $3.4(2.9) *$ & $4.2(2.8) * S$ \\
\hline \multicolumn{9}{|l|}{ Countermeasures } \\
\hline Optimism & $3.0(1.0)$ & $2.9(1.1)$ & $2.5(1.2)$ & $2.7(I . I)$ & $3.3(0.7)$ & $3.1(1.0)$ & $3.0(1.0) *$ & $2.8(1.1) *$ \\
\hline Coping with stress & $6.3(1.5)$ & $5.6(1.8)$ & $5.8(1.8)$ & $5.3(1.7)$ & $6.6(1.5)$ & $5.7(1.8)^{\mathrm{S}}$ & $6.3(1.7)$ & $5.6(1.9)^{\mathrm{S}}$ \\
\hline Support of family and friends & $4.7(2.1)$ & $5.8(1.8)$ & $4.8(2.0)$ & $6.0(1.6)$ & $6.1(2.1)^{\dagger}$ & $6.6(1.7)^{\dagger}$ & $6.3(1.8)^{\dagger}$ & $6.6(1.6)$ \\
\hline Nutrition & $6.8(3.1)$ & $7.4(2.9)$ & $6.7(2.9)$ & $7.3(2.7)$ & $7.5(3.1)$ & $8.0(2.7)$ & $7.8(2.6)$ & $7.4(2.9) *$ \\
\hline Being active & $6.5(2.5)$ & $6.9(2.1)$ & $5.2(2.2) *$ & $5.8(2.8)$ & $7.0(2.2)$ & $6.8(2.3)$ & $5.9(2.6) *$ & $5.7(2.5) *$ \\
\hline Physical activity level & $5.5(2.2)$ & $5.5(1.7)$ & $4.7(2.2)$ & $4.9(2.2)$ & $5.0(2.0)$ & $5.3(2.1)$ & $4.9(2.0)$ & $4.6(2.0) *$ \\
\hline Sleep & $3.0(1.0)$ & $2.7(1.1)$ & $2.6(1.1)$ & $2.6(1.1)$ & $3.0(0.9)$ & $2.7(1.0)$ & $3.1(0.9)$ & $2.6(1.1)^{\mathrm{s}}$ \\
\hline \multicolumn{9}{|l|}{ Health correlates } \\
\hline Quality of life & $7.0(2.1)$ & $7.4(1.6)$ & $5.6(2.4) *$ & $6.2(2.3) *$ & $7.6(1.5)$ & $7.6(1.7)$ & $6.6(1.9) * t$ & $6.6(2.0) *$ \\
\hline Perceived immune fitness & $7.6(1.1)$ & $7.4(1.8)$ & $7.2(1.2)$ & $7.1(2.2)$ & $7.9(1.4)$ & $7.3(1.9)^{\mathrm{s}}$ & $7.7(1.5)^{\dagger}$ & $7.1(2.0)^{\mathrm{S}}$ \\
\hline COVID-19 Symptom - presence & $2.1(2.0)$ & $2.8(2.2)$ & $2.3(2.0)$ & $3.3(2.3)$ & $2.7(2.1)$ & $2.6(2.2)$ & $2.4(2.0)$ & $2.5(2.2)$ \\
\hline COVID-19 Symptom - severity & $2.7(2.9)$ & $3.9(3.9)$ & $3.2(3.9)$ & $4.9(4.7)$ & $3.5(3.4)$ & $3.8(4.0)$ & $3.1(3.2)$ & $3.8(4.0)$ \\
\hline
\end{tabular}

Notes: Mean and standard deviation (SD, between brackets) are shown. Significant sex differences are indicated by ${ }^{\mathrm{S}}$. Differences for mood and countermeasures were considered significant if $\mathrm{p}<0.00625$, after Bonferroni corrections for multiple comparisons, and if $\mathrm{p}<0.05$ for health correlates. Significant differences between before and during lockdown are indicated by*; significant differences between the living alone group and living together group are indicated by ${ }^{\dagger}$.

Interestingly, loneliness increased for both groups. This suggests that the feeling of being lonely may not be wholly dependent on social connectedness but also contain a psychological component. Despite living together, one may still feel isolated from other people, and perhaps some may lack their usual social support from people they are not residing with, and therefore feeling disconnected from (the social side of) normal life. Additionally, poor family/ relationship functioning may also contribute to loneliness, despite being surrounded by them. ${ }^{3}$ In line with other studies, we found that loneliness was associated with reduced feelings of happiness, increased ratings of depression and stress, and a decreased quality of life. ${ }^{3,6}$

Results indicate that despite both groups experiencing increases in feelings of loneliness, those living alone have significantly larger increases in these feelings compared to those living together. The implementation of various restrictions, such as the limited number of guests allowed to visit one's home and advised stay-at-home orders, may have been particularly difficult for those living alone as this naturally hinders physical social interactions. For these people, contact with others may be made through various social media platforms. Despite the availability thereof, one study conducted in the United States noted that contact via the phone did not prove to have significant positive effects. In fact, results highlighted that contact via the phone may even increase feelings of loneliness as it can remind someone that they are alone. This emphasizes the importance of physical interaction for one's emotional wellbeing. ${ }^{37}$ However, this study only looked at the effects of telephone contact, and not at contact via other forms of social media. Nevertheless, the above-mentioned points suggest that those living alone may be particularly reliant on others for social interaction and support, therefore 
being more sensitive to social distancing measures, and explaining why higher rates of loneliness were observed amongst those living alone. ${ }^{3}$

Furthermore, results show that these feelings of loneliness are accompanied by greater increases in reported COVID-19-related symptoms for the group that lives alone. Also, negative correlations of perceived immune fitness with anxiety and loneliness were significantly stronger for those living alone. Numerous studies have shown that there are reliable correlations between feelings of loneliness and psychological symptoms, such as anxiety, depression, and stress. Moreover, feelings of loneliness may not only be a consequence of stress but also act as a cause, creating a vicious cycle. It has been found that these psychological effects can negatively impact immune fitness, as it leads to activation of the HPA-axis, increasing levels of cortisol. As social factors, such as social isolation and loneliness, can lead to glucocorticoid resistance, this removes the natural break-mechanism of cortisol production, further aggravating levels of stress, as well as stressinduced depression and anxiety. Moreover, loneliness has been associated with decreases in the number of immune cells, especially decreases in the ratios of neutrophil to monocyte and neutrophil to lymphocyte ratios. These decreases have been observed in direct proportion to circulating cortisol ratios, supporting earlier research claiming a relationship between stress and a decrease in immune functioning. For this reason, those living alone and experiencing greater rates of loneliness and anxiety may report larger increases in COVID-19 related symptoms. $3,6,10,13$

Decreases in perceived immune fitness may be the result of an overall decrease in mood. ${ }^{36}$ As mentioned, social support can be protective against feelings of loneliness, leading to decreases in feelings of stress and anxiety. ${ }^{38}$ As no effect was seen on COVID-19 symptom severity and presence for those living together, this may suggest that the overall effect of social support may weigh heavier on one's wellbeing than overall mood. Therefore, social support resulting from living together with others may play a fundamental role in regulating mood and perceived immune fitness, highlighting the importance of social support in one's surroundings.

Our data show positive correlations for both groups between being active, optimistic, being able to cope with stress and improved perceived immune fitness, indicating these assets have a beneficial effect for someone's health. Physical exercise is known to play a key role in regulating the immune system and reducing inflammatory markers in the body. Mechanisms may include stimulation of various immune cells, such as neutrophils and cytokines immunoglobulins, resulting in greater ability for the immune system to react to pathogens. Therefore, exercise may improve immune fitness and can potentially help to decrease the likelihood of acquiring the COVID-19 virus. $^{39,40}$

On the contrary, research has shown that those having developed a more sedentary lifestyle due to the pandemic can increase susceptibility to infections. Additionally, physical activity has been shown to reduce feelings of anxiety, depression, and stress, perhaps contributing to overall optimism and ability to cope with stress. ${ }^{39}$

Several limitations of the current study should be considered. First, the current study relied on retrospective survey data, which may be subject to recall bias, and could perhaps be subject to either over- or underestimating feelings accompanying lockdown. This could potentially have had an impact on the accuracy of the data. Secondly, perceived immune fitness was assessed using a subjective rating scale, and while this method has been used in other studies, it is possible that the subjective perception of immune fitness may be different from objective biomarker assessments (eg, cytokines). In this context, it is also relevant to mention that individuals may differ in health (risk) perception and the involvement of sensory perception in emotional processes. Several factors may influence perception, including alexithymia. ${ }^{41}$ Research in the context of COVID-19 revealed that risk perception plays an important role in determining to what extent mental health is affected during the COVID-19 pandemic, ${ }^{42}$ whereas another study revealed that the factors, age, sex, and marital status, were significant predictors of the perceived risk of getting a disease caused by a novel virus. ${ }^{43}$

The COVID-19 symptoms scale used was created at the beginning of the lockdown period in the Netherlands in 2020 and since then, the Dutch National Institute for Public Health and Environment (RIVM) has included additional symptoms to the list of COVID-19 symptoms. As such, these symptoms were not included in our scale and must be included in further research. Additionally, not all respondents have been tested for COVID-19, and therefore it is unknown whether this subsample has been previously infected with COVID-19. Thus, although there was a significant correlation between living alone and COVID-19 symptoms, further research should be done to confirm these findings. Moreover, since this study was 
cross-sectional and no longitudinal follow-up has been conducted yet, it is not possible to compare the collected data with that of the following summer period (without lockdown), and second lockdown period (November 2020 - April 2021) that followed. Long-term effects of the pandemic resulting from living together or alone may be crucial to determine the overall effects the pandemic has had on individuals, as well as being useful for the future. It may be possible that certain effects may not be sustained over time, or alternatively, that the effects will be much more pronounced for the second lockdown, which was of longer duration. Finally, participants were recruited via Facebook, limiting respondents to those with an internet connection and a Facebook account. Furthermore, more women responded than men to the survey, and the majority of the sample did not live alone during the lockdown. For the above-mentioned reasons, it is unclear to what extent the results of the survey are representative of the entire Dutch population.

Notwithstanding these limitations, the survey revealed that living alone is associated with more negative effects on mood, perceived immune fitness and experiencing COVID-19 symptoms than living together with others. The data show that the two groups differ significantly in the level of support for family and friends, which is lower in people who lived alone during lockdown. The findings imply that enhancing social support and social interactions in people that live alone may contribute to a better mental resilience and ability to cope with the negative effects of lockdown.

\section{Conclusion}

This study examined the impact of living alone or together during the first lockdown period in the Netherlands, and the consequent effects on mood, perceived immune fitness and experiencing of COVID-19 symptoms. Our results are consistent with other studies, indicating those living alone were more susceptible to feelings of depression and loneliness, feelings that corresponded with a decrease in perceived immune fitness, and differentially experiencing COVID-19 symptoms. In contrast, people who lived together during lockdown reported significantly greater support from family and friends, and less pronounced mood changes. These outcomes suggest that adequate social support and interpersonal contacts are vital for one's health, in particularly during a global pandemic.

\section{Disclosure}

This research received no external funding. Over the past 3 years, J.C.V. has acted as a consultant/advisor for KNMP, More Labs, Red Bull, Sen-Jam Pharmaceutical, Toast!, Tomo, and ZBiotics. J.G. is a part-time employee of Nutricia Research and has received research grants from Nutricia Research Foundation, Top Institute Pharma, Top Institute Food and Nutrition, GSK, STW, NWO, Friesland Campina, CCC, Raak-Pro, and the EU. The other authors have no potential conflicts of interest to disclose.

\section{References}

1. Adil MT, Rahman R, Whitelaw D, et al. SARS-CoV-2 and the pandemic of COVID-19. Postgrad Med J. 2021;97(1144):110-116. PMID: 32788312. doi:10.1136/postgradmedj-2020-138386

2. Daly Z, Slemon A, Richardson CG, et al. Associations between periods of COVID-19 quarantine and mental health in Canada. Psychiatry Res. 2021;295:113631. PMID: 33310417; PMCID: PMC7718586. doi:10.1016/j.psychres.2020.113631

3. Wong SYS, Zhang D, Sit RWS, et al. Impact of COVID-19 on loneliness, mental health, and health service utilisation: a prospective cohort study of older adults with multimorbidity in primary care. $\mathrm{Br} J$ Gen Pract. 2020;70(700):e817-e824. PMID: 32988955; PMCID: PMC7523921. doi:10.3399/bjgp20X713021

4. Kämpfen F, Kohler IV, Ciancio A, Bruine de Bruin W, Maurer J, Kohler HP. Predictors of mental health during the Covid-19 pandemic in the US: role of economic concerns, health worries and social distancing. PLoS One. 2020;15(11):e0241895. PMID: 33175894; PMCID: PMC7657497. doi:10.1371/journal.pone.0241895

5. Torales J, O'Higgins M, Castaldelli-Maia JM, Ventriglio A. The outbreak of COVID-19 coronavirus and its impact on global mental health. Int J Soc Psychiatry. 2020;66(4):317-320. PMID: 32233719. doi:10.1177/0020764020915212

6. Campagne DM. Stress and perceived social isolation (loneliness). Arch Gerontol Geriatr PMID: 30825769. 2019;82:192-199. doi:10.1016/j.archger.2019.02.007

7. Mudrazija S, Angel JL, Cipin I, Smolic S. Living alone in the United States and Europe: the impact of public support on the Independence of older adults. Res Aging. 2020;42(5-6):150-162. PMID: 32116111. doi:10.1177/0164027520907332

8. Robb CE, de Jager CA, Ahmadi-Abhari S, et al. Associations of social isolation with anxiety and depression during the early COVID-19 pandemic: a survey of older adults in London, UK. Front Psychiatry. 2020;11:591120. PMID: 33132942; PMCID: PMC7566017. doi:10.3389/fpsyt.2020.591120

9. Cabello M, Izquierdo A, Leal I. Loneliness and not living alone is what impacted on the healthcare professional's mental health during the COVID-19 outbreak in Spain. Health Soc Care Community. 2021. PMID: 33761161. doi:10.1111/hsc. 13260

10. Luchetti M, Lee JH, Aschwanden D, et al. The trajectory of loneliness in response to COVID-19. Am Psychol. 2020;75(7):897-908. PMID: 32567879; PMCID: PMC7890217. doi:10.1037/amp0000690

11. Kamin T, Perger N, Debevec L, Tivadar B. Alone in a time of pandemic: solo-living women coping with physical isolation. Qual Health Res. 2021;31(2):203-217. PMID: 33213283. doi:10.1177/ 1049732320971603

12. Delmastro M, Zamariola G. Depressive symptoms in response to COVID-19 and lockdown: a cross-sectional study on the Italian population. Sci Rep. 2020;10(1):22457. PMID: 33384427; PMCID: PMC7775443. doi:10.1038/s41598-020-79850-6 
13. Lahav Y. Psychological distress related to COVID-19 - The contribution of continuous traumatic stress. $J$ Affect Disord. 2020;277:129-137. PMID: 32818776; PMCID: PMC7416772. doi:10.1016/j.jad.2020.07.141

14. Luo M, Guo L, Yu M, Jiang W, Wang H. The psychological and mental impact of coronavirus disease 2019 (COVID-19) on medical staff and general public - A systematic review and meta-analysis. Psychiatry Res. 2020;291:113190. PMID: 32563745; PMCID: PMC7276119. doi:10.1016/j.psychres.2020.113190

15. Bhatti AB, Haq AU. The pathophysiology of perceived social isolation: effects on health and mortality. Cureus. 2017;9(1):e994. PMID: 28382237; PMCID: PMC5367921. doi:10.7759/cureus.994

16. Kempuraj D, Selvakumar GP, Ahmed ME, et al. COVID-19, Mast cells, cytokine storm, psychological stress, and neuroinflammation. Neuroscientist. $\quad$ 2020;26(5-6):402-414. PMID: 32684080. doi: $10.1177 / 1073858420941476$

17. Chew QH, Chia FL, Ng WK, et al. Perceived stress, stigma, traumatic stress levels and coping responses amongst residents in training across multiple specialties during COVID-19 pandemic A longitudinal study. Int J Environ Res Public Health. 2020;17 (18):6572. PMID: 32916996; PMCID: PMC7559162. doi:10.3390/ ijerph 17186572

18. Evans S, Mikocka-Walus A, Klas A, et al. From "It has stopped our lives" to "Spending more time together has strengthened bonds": the varied experiences of Australian families during COVID-19. Front Psychol. 2020;11:588667. PMID: 33192922; PMCID: PMC7606874. doi:10.3389/fpsyg.2020.588667

19. Cansel N, Ucuz İ, Arslan AK, et al. Prevalence and predictors of psychological response during immediate COVID-19 pandemic. Int J Clin Pract. 2021;75(5):e13996. PMID: 33404167; PMCID: PMC7883178. doi:10.1111/ijcp.13996

20. Choi EY, Farina MP, Wu Q, Ailshire J. COVID-19 Social distancing measures and loneliness among older adults. J Gerontol B Psychol Sci Soc Sci. 2021:gbab009. PMID: 33428753; PMCID: PMC7928665. doi: 10.1093/geronb/gbab009

21. Mari E, Fraschetti A, Lausi G, et al. Forced Cohabitation during coronavirus lockdown in Italy: a study on coping, stress and emotions among different family patterns. J Clin Med. 2020;9(12):3906. PMID: 33272002; PMCID: PMC7761111. doi:10.3390/jcm9123906

22. Baumeister RF, Leary MR. The need to belong: desire for interpersonal attachments as a fundamental human motivation. Psychol Bull. 1995;117(3):497-529. PMID: 7777651. doi:10.1037/00332909.117.3.497

23. Amati V, Meggiolaro S, Rivellini G, Zaccarin S. Social relations and life satisfaction: the role of friends. Genus. 2018;74(1):7. PMID: 29755134; PMCID: PMC5937874. doi:10.1186/s41118-018-0032-z

24. Duan H, Yan L, Ding X, Gan Y, Kohn N, Wu J. Impact of the COVID-19 pandemic on mental health in the general Chinese population: changes, predictors and psychosocial correlates. Psychiatry Res. 2020;293:113396. PMID: 32861096; PMCID: PMC7431364. doi:10.1016/j.psychres.2020.113396

25. Kiani P, Merlo A, Saeed HM, et al. Immune fitness and the psychosocial and health consequences of the COVID-19 pandemic lockdown in The Netherlands: methodology and design of the CLOFIT study. Eur J Invest Health Psychol Educ. 2021;11:199-218.

26. Wilod Versprille LJF, van de Loo AJAE, Mackus M, et al. Development and validation of the Immune Status Questionnaire (ISQ). Int J Environ Res Public Health. 2019;16(23):pii: E4743. doi:10.3390/ijerph16234743

27. Verster JC, Sandalova E, Garssen J, Bruce G. The use of single-item ratings versus traditional multiple-item questionnaires to assess mood and health. Eur J Invest Health Psychol Educ. 2021;11:183-198.

28. Wilson DMC, Nielsen E, Ciliska D. Lifestyle assessment: testing the FANTASTIC Instrument. Can Fam Physician. 1984;30:1863-1866.
29. Sharratt JK, Sharratt MT, Smith DM, Howell NJ, Davenport L. FANTASTIC Lifestyle survey of University of Waterloo employees. Can Fam Physician. 1984;30:1869-1872.

30. Van Schrojenstein Lantman M, Otten LS, Mackus M, et al. Mental resilience, perceived immune functioning, and health. $J$ Multidiscip Healthc. 2017;10:107-112. doi:10.2147/JMDH.S130432

31. Gopal A, Sharma AJ, Subramanyam MA. Dynamics of psychological responses to COVID-19 in India: a longitudinal study. PLoS One. 2020;15(10):e0240650. doi:10.1371/journal.pone.0240650

32. Landi G, Pakenham KI, Boccolini G, Grandi S, Tossani E. Health anxiety and mental health outcome during COVID-19 lockdown in Italy: the mediating and moderating roles of psychological flexibility. Front Psychol. 2020;11:2195. doi:10.3389/fpsyg.2020.02195

33. Biviá-Roig G, La Rosa VL, Gómez-Tébar M, et al. Analysis of the impact of the confinement resulting from COVID-19 on the lifestyle and psychological wellbeing of Spanish Pregnant women: an internet-based cross-sectional survey. Int $J$ Environ Res Public Health. 2020;17(16):5933. doi:10.3390/ijerph17165933

34. Commodari E, La Rosa VL. Adolescents in quarantine during COVID-19 pandemic in Italy: perceived health risk, beliefs, psychological experiences and expectations for the future. Front Psychol. 2020;11:559951. doi:10.3389/fpsyg.2020.559951

35. Hossain MM, Tasnim S, Sultana A, et al. Epidemiology of mental health problems in COVID-19: a review. F1000Res. 2020;9:636. PMID: 33093946; PMCID: PMC7549174. doi:10.12688/ f1000research.24457.1

36. Raj T, Bajaj A. Living alone in lockdown: impact on mental health and coping mechanisms among young working adults. Curr Psychol. 2021:1-13. PMID: 33758485; PMCID: PMC7972330. doi: 10.1007/ s12144-021-01511-2

37. Fingerman $\mathrm{KL}, \mathrm{Ng} \mathrm{YT}$, Zhang $\mathrm{S}$, et al. Living alone during COVID-19: social contact and emotional wellbeing among older adults. J Gerontol B Psychol Sci Soc Sci. 2021;76(3):e116-e121. doi:10.1093/geronb/gbaa200 PMID: 33196815; PMCID: PMC7717423.

38. Ruthig JC, Hanson BL, Pedersen H, Weber A, Chipperfield JG. Later life health optimism, pessimism and realism: psychosocial contributors and health correlates. Psychol Health. 2011;26(7):835-853. PMID: 21432733. doi:10.1080/08870446.2010.506574

39. Abdelbasset WK. Stay home: role of physical exercise training in elderly individuals' ability to face the COVID-19 infection. J Immunol Res. 2020;2020:8375096. PMID: 33354578; PMCID: PMC7737455. doi:10.1155/2020/8375096

40. Ravalli S, Musumeci G. Coronavirus outbreak in Italy: physiological benefits of home-based exercise during pandemic. J Funct Morphol Kinesiol. 2020;5(2):31. PMID: 33467247; PMCID: PMC7739427. doi:10.3390/jfmk5020031

41. Serafini G, Gonda X, Pompili M, Rihmer Z, Amore M, Engel-Yeger $B$. The relationship between sensory processing patterns, alexithymia, traumatic childhood experiences, and quality of life among patients with unipolar and bipolar disorders. Child Abuse Neglect. 2016;62:39-50. doi:10.1016/j.chiabu.2016.09.013

42. Ding Y, Xu J, Huang S, Li P, Lu C, Xie S. Risk perception and depression in public health crises: evidence from the COVID-19 crisis in China. Int J Environ Res Public Health. 2020;17(16):5728. doi:10.3390/ijerph17165728

43. Commodari E, La Rosa VL, Coniglio MA. Health risk perceptions in the era of the new coronavirus: are the Italian people ready for a novel virus? A cross-sectional study on perceived personal and comparative susceptibility for infectious diseases. Public Health. 2020;187:8-14. doi:10.1016/j.puhe.2020.07.036 


\section{Publish your work in this journal}

Psychology Research and Behavior Management is an international, peer-reviewed, open access journal focusing on the science of psychology and its application in behavior management to develop improved outcomes in the clinical, educational, sports and business arenas. Specific topics covered in the journal include: Neuroscience, memory and decision making; Behavior modification and management; Clinical

applications; Business and sports performance management; Social and developmental studies; Animal studies. The manuscript management system is completely online and includes a very quick and fair peer-review system, which is all easy to use. Visit http://www. dovepress.com/testimonials.php to read real quotes from published authors. 